\title{
Single-Spin Addressing in an Atomic Mott Insulator
}

\author{
Christof Weitenberg ${ }^{1}$, Manuel Endres ${ }^{1}$, Jacob F. Sherson ${ }^{1 \dagger}$, Marc Cheneau ${ }^{1}$, \\ Peter Schauß $\beta^{1}$, Takeshi Fukuhara ${ }^{1}$, Immanuel Bloch ${ }^{1,2}$, and Stefan Kuhr ${ }^{1 *}$ \\ ${ }^{1}$ Max-Planck-Institut für Quantenoptik, Hans-Kopfermann-Str. 1, 85748 Garching, Germany \\ ${ }^{2}$ Ludwig-Maximilians-Universität, Schellingstr. 4/II, 80799 München, Germany
}

(Dated: 9 January 2011)

\begin{abstract}
Ultracold atoms in optical lattices are a versatile tool to investigate fundamental properties of quantum many body systems. In particular, the high degree of control of experimental parameters has allowed the study of many interesting phenomena such as quantum phase transitions and quantum spin dynamics. Here we demonstrate how such control can be extended down to the most fundamental level of a single spin at a specific site of an optical lattice. Using a tightly focussed laser beam together with a microwave field, we were able to flip the spin of individual atoms in a Mott insulator with sub-diffraction-limited resolution, well below the lattice spacing. The Mott insulator provided us with a large two-dimensional array of perfectly arranged atoms, in which we created arbitrary spin patterns by sequentially addressing selected lattice sites after freezing out the atom distribution. We directly monitored the tunnelling quantum dynamics of single atoms in the lattice prepared along a single line and observed that our addressing scheme leaves the atoms in the motional ground state. Our results open the path to a wide range of novel applications from quantum dynamics of spin impurities, entropy transport, implementation of novel cooling schemes, and engineering of quantum many-body phases to quantum information processing.
\end{abstract}

The ability to observe and control the position of single atoms on a surface of a solid via scanning tunnelling and atomic force microscopy has revolutionised the field of condensed matter physics [1, 2]. In few-atom systems, coherent control of single particles in e.g. an ion chain has proven crucial for the implementation of high-fidelity quantum gates and the readout of individual qubits in quantum information processing [3]. Bringing such levels of control to the regime of large scale many-body systems has been a longstanding goal in quantum physics. In the context of ultracold atoms in optical lattices, a major challenge has been to combine degenerate atomic samples with single-site addressing resolution and singleatom sensitivity. This full control is essential for many applications in condensed matter physics, such as the study of spin impurities [4] and quantum spin dynamics [5], 6] within quantum magnetism, entropy transport, the implementation of novel cooling schemes [7, 8, or digital quantum simulations based on Rydberg atoms [9]. For scalable quantum information processing, a Mott insulator with unity filling provides a natural quantum register with several hundreds of qubits. In order to exploit the full potential of such a large scale system for quantum computation, coherent manipulation of individual spins is indispensable, both within a circuit-based [10] or a one-way quantum computer architecture [11, 12].

The quest to address atoms on single sites of an optical lattice has a long history [7, 13, 22. In one dimension, single-site addressing was accomplished optically

\footnotetext{
$\dagger$ present address: Department of Physics and Astronomy, University of Aarhus, DK-8000 Aarhus C, Denmark.

* Electronic address: stefan.kuhr@mpq.mpg.de
}

in a long-wavelength lattice [23, in which however tunnelling was completely suppressed, and using magnetic resonance techniques in a sparsely filled short-wavelength lattice [24, 25. In two dimensions, an electron beam was used to depopulate sites of a Bose-Einstein condensate loaded into an array of potential tubes, each containing up to 80 atoms [26. In this case, coherent spin manipulation was not possible and the readout was done by averaging over more than 100 single images. None of the experiments so far has shown single-atom spin control in strongly correlated systems together with high fidelity single-atom detection.

Here we report on the achievement of this goal, by demonstrating single-site-resolved addressing and control of the spin states of individual atoms in a Mott insulator in an optical lattice. The Mott insulator provided us with an almost perfect initial two-dimensional array of atoms in the same spin state. Apart from few thermal defects, each lattice site contained a single atom in its motional ground state [27, 28]. Using a tightly focused laser beam, we introduced a controlled differential energy shift between two atomic spin states at a given lattice site. Microwave radiation resonant with this shifted transition then allowed us to selectively address the spin of a single atom 7, 17 with high fidelity. We thereby obtained sub-diffraction-limited spatial resolution well below the lattice spacing. By moving the addressing laser beam to different lattice sites and by inducing spin-flips in the Mott insulator, we were able to deterministically create arbitrary two-dimensional spin patterns of individual atoms, thereby realising a scalable single-atom source [29 31]. Furthermore, we investigated how much our single-spin manipulation affects the motional state of the atoms by directly monitoring the tunnelling dynamics of single atoms after addressing them. Averaging over 
a

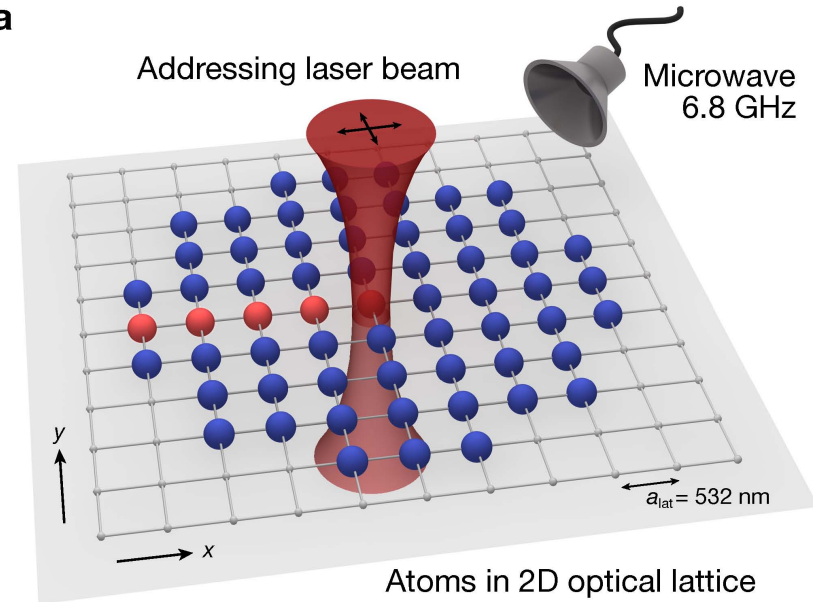

b



FIG. 1: Addressing scheme. a, Atoms in a Mott insulator with unity filling arranged on a square lattice with period $a_{\text {lat }}=532 \mathrm{~nm}$ were addressed using an off-resonant laser beam. The beam was focussed onto individual lattice sites by a high-aperture microscope objective (not shown) and could be moved in the $x y$ plane with an accuracy of better than $0.1 a_{\text {lat }}$. b, Energy diagram of atoms in the lattice for the two hyperfine states $|0\rangle$ and $|1\rangle$. The $\sigma^{-}$-polarized addressing beam locally induces a light shift $\Delta_{\mathrm{LS}}$ of state $|1\rangle$, bringing it into resonance with a microwave field. A Landau-Zener sweep (central frequency $\omega_{\mathrm{MW}}$, sweep width $\sigma_{\mathrm{MW}}$ ) transfers the addressed atoms from $|0\rangle$ to $|1\rangle$.

several snapshots after different tunnelling times, we fully reconstructed the characteristic spatial probability distribution of the single-atom wave function and its coherent evolution over more than 20 lattice sites. We were able to discriminate the dynamics of the atoms in the zeroth and in the first band and found that most of the atoms remained in the motional ground state after addressing.

\section{Experimental setup}

In our experiments, we prepared a two-dimensional sample of ultracold ${ }^{87} \mathrm{Rb}$ atoms in an optical lattice, confined in a single antinode of a vertical standing wave along the $z$ direction. Two pairs of counterpropagating laser beams (wavelength $\lambda=1064 \mathrm{~nm}$ ) along the horizontal $x$ and $y$ directions provided a square lattice with period of $a_{\text {lat }}=\lambda / 2=532 \mathrm{~nm}$ (for details see Ref. [28]). Starting from a Bose-Einstein condensate we raised the potential in the $x$ and $y$ lattice axes within $75 \mathrm{~ms}$ to values of $V_{x, y}=23(2) E_{r}$ (the number in parenthesis denotes the $1 \sigma$ uncertainty of the last digit), where $E_{r}=h^{2} /\left(2 m \lambda^{2}\right)$ is the recoil energy, and $m$ denotes the atomic mass of ${ }^{87} \mathrm{Rb}$. In this way, the interaction energy of the particles with respect to their kinetic energy was increased such that the system undergoes a quantum phase transition to a Mott insulating state [32 34]. Due to the external harmonic confinement, the Mott insulator exhibits a shell structure with fixed integer atom numbers increasing in a step-like manner from the outer regions of the system to the inner core [35, 36. The initial state for all experiments presented in this paper was a single shell with only one atom per lattice site, which in our system was realised for atom numbers smaller than $\sim 400$.

We detected the atoms using fluorescence imaging via a high-resolution microscope objective with numerical aperture of NA $=0.68$. An optical molasses induced fluorescence light and simultaneously laser-cooled the atoms. Light-assisted collisions lead to rapid losses of atom pairs, such that we only detected the atom number modulo two on each lattice site [27, 28]. With about 5,000 collected photons per atom, we identified individual atoms in the lattice with an excellent signal-to-noise ratio. Even in the regions of high atomic density, we determined the presence or absence of an atom for each lattice site with $>99.5 \%$ fidelity using a special reconstruction algorithm [28].

\section{Addressing single lattice sites}

In order to address the atoms in the lattice, we used an off-resonant laser beam focused by the high-resolution imaging system onto individual lattice sites (Fig. 1). The laser beam causes a differential light shift of the two relevant hyperfine levels $|0\rangle \equiv\left|F=1, m_{F}=-1\right\rangle$ and $|1\rangle \equiv\left|F=2, m_{F}=-2\right\rangle$ and tunes the addressed atom into resonance with an external microwave field at $\sim 6.8$ GHz. The $\sigma^{-}$-polarized addressing beam had a wavelength of $787.55 \mathrm{~nm}$, between the $D_{1}$ and $D_{2}$ lines, in order to obtain a large differential light shift between the two hyperfine levels. For perfect $\sigma^{-}$-polarization, this 'magic' wavelength generates a light shift only for state $|1\rangle$, and leaves state $|0\rangle$ unaffected. The beam had a diameter of $\sim 600 \mathrm{~nm}$ full-width at half-maximum (FWHM) and could be moved in the object plane over the entire field of view by changing its angle of incidence into the microscope objective with a two-axis piezo mirror. We were able to position the beam with an accuracy better than $0.1 a_{\text {lat }}$ using an independent calibration 

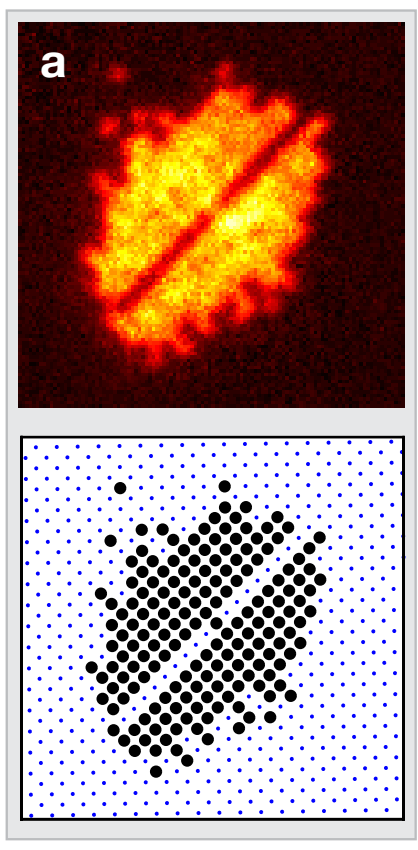
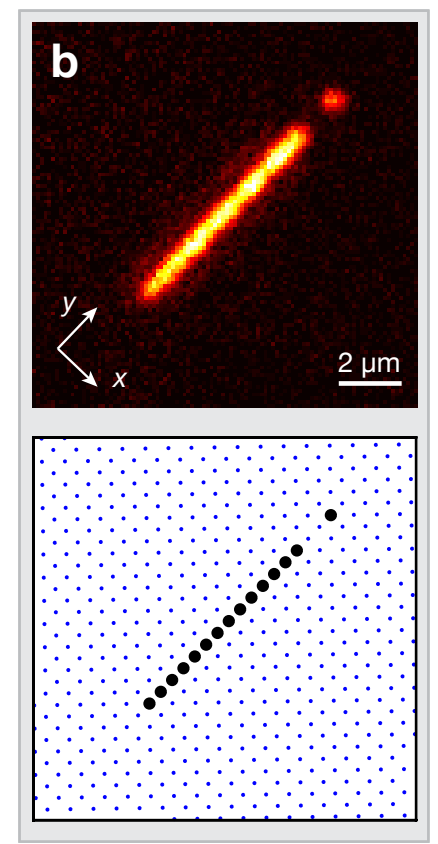
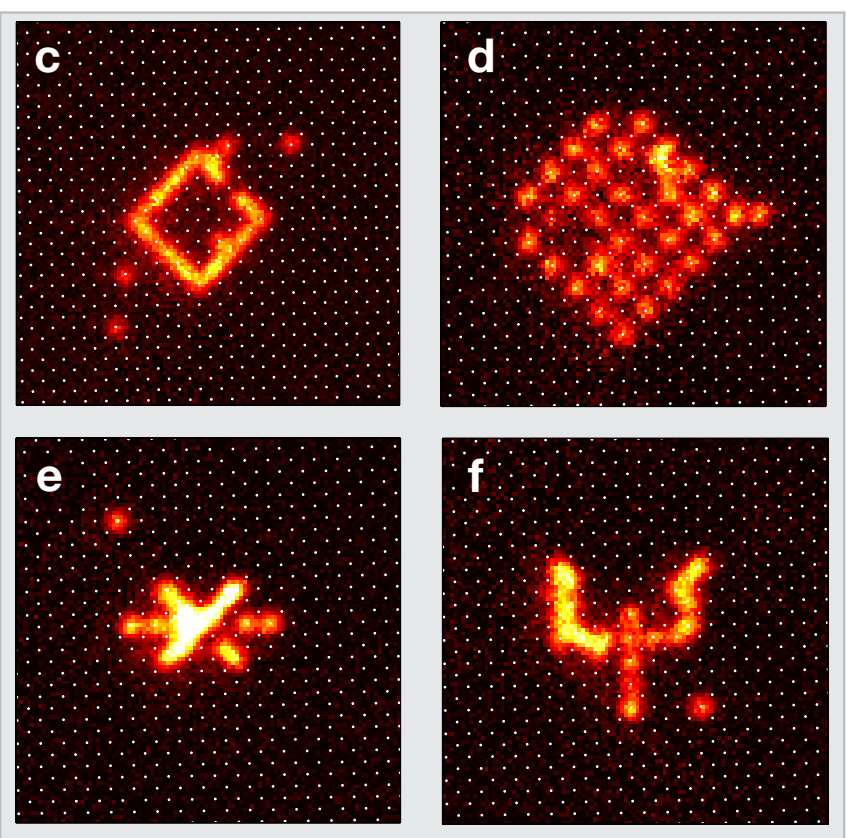

FIG. 2: Single-site addressing. a, Experimentally obtained fluorescence image of a Mott insulator with unity filling in which the spin of selected atoms was flipped from $|0\rangle$ to $|1\rangle$ using our single-site addressing scheme. Atoms in state $|1\rangle$ were removed by a resonant laser pulse before detection. The lower part shows the reconstructed atom number distribution on the lattice. Each circle indicates a single atom, the points mark the lattice sites. b-f, Same as a, but a global microwave sweep exchanged the population in $|0\rangle$ and $|1\rangle$, such that only the addressed atoms were observed. The line in $\mathbf{b}$ shows 14 atoms on neighbouring sites, the images c-f contain 29, 35, 18 and 23 atoms, respectively. The single isolated atoms in b,e,f were placed intentionally to allow for the correct determination of the lattice phase for the feedback on the addressing beam position.

measurement of its position together with a feedback that tracks the slowly varying lattice phases (see Appendix).

If the addressing laser beam is perfectly centered onto one lattice site (see Fig.1 $\mathrm{b}$ ), the differential light shift is $\Delta_{\mathrm{LS}} /(2 \pi) \simeq-70 \mathrm{kHz}$, whereas a neighbouring atom only experiences $10 \%$ of the peak intensity. The resulting difference in light shifts can be well resolved spectrally by our microwave pulses. In order to flip the spin, we performed Landau-Zener sweeps (see Appendix) of $\sigma_{\mathrm{MW}} /(2 \pi)=60 \mathrm{kHz}$ width and $20 \mathrm{~ms}$ duration yielding a near flat-top frequency spectrum with a maximum population transfer efficiency of $\sim 95 \%$.

As a first experiment, we sequentially flipped the spin of the atoms at selected lattice sites in our Mott insulator with unity filling and spin state $|0\rangle$ (Fig. 22 ). The lattice depths were first changed to $V_{x}=56 E_{r}, V_{y}=90 E_{r}$ and $V_{z}=70 E_{r}$ in order to completely suppress tunnelling even when the addressing beam locally perturbs the lattice potential. For each lattice site, we then switched on the addressing laser beam with an s-shaped ramp within $2.5 \mathrm{~ms}$, which is adiabatic with respect to the on-site oscillation frequency of $\sim 30 \mathrm{kHz}$. Subsequently, a microwave pulse with the parameters described above produced spin-flips from $|0\rangle$ to $|1\rangle$. The addressing laser was switched off again within $2.5 \mathrm{~ms}$, before its position was changed in $5 \mathrm{~ms}$ to address the next lattice site. For the image of Fig. $2 \mathrm{a}$, this procedure was repeated 16 times in order to flip the spins along a line. Finally, a $5 \mathrm{~ms}$ 'pushout' laser pulse, resonant with the $F=2$ to $F^{\prime}=3$ transition, removed all addressed atoms in state $|1\rangle$. In order to reveal only the spin-flipped atoms, the spin states of all atoms were flipped by a global microwave sweep before the push-out laser was applied (Fig.22,d). However, due to the finite transfer efficiency of the global sweep, some atoms remaining in state $|0\rangle$ were clearly visible in addition to the addressed ones. To avoid this problem when detecting the addressed atoms, we initially transferred the whole sample to state $|1\rangle$ by a microwave sweep and then shone in a repumping laser that completely depopulated state $|0\rangle$. Then, we used our addressing scheme to transfer selected atoms back to $|0\rangle$ and subsequently pushed out the atoms in $|1\rangle$, yielding typical images as shown in Fig.2b,e,f.

\section{Spin-flip fidelity}

We quantified the success rate of our addressing scheme by again producing a series of spin-flips along the $y$ lattice axis in a Mott insulator with unity filling (see Fig. 3). The experimental procedure was the same as described above for the realisation of Fig. 2 a, in which the addressed sites were detected as empty sites. From the reconstructed atom number distribution (Fig.3 3 ), we determined the 


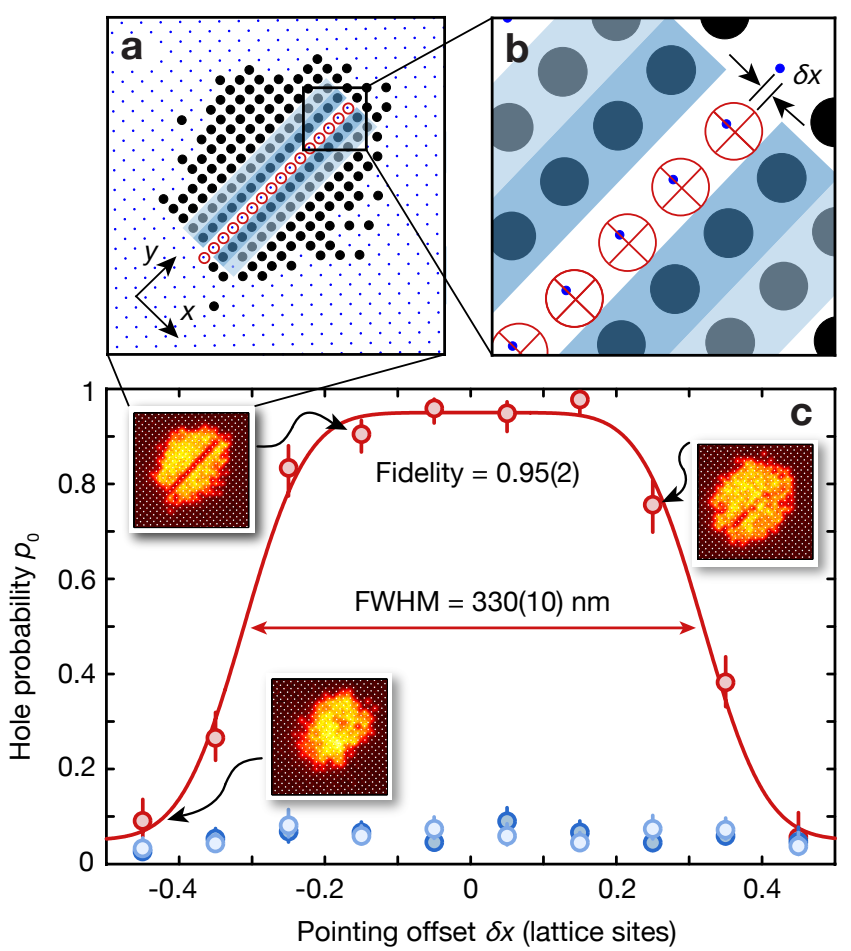

FIG. 3: Addressing fidelity. The spin-flip probability was measured by sequentially addressing a series of 16 neighbouring sites along the $y$ lattice axis (red circles in a) in a Mott insulator with unity filling. The red data points in $\mathbf{c}$ show the resulting hole probability $p_{0}(\delta x)$ as a function of the pointing offset $\delta x$, as defined in $\mathbf{b}$. Each point was obtained by averaging over $4-7$ pictures (total $50-100$ addressed lattice sites), taking only those sites into account which lie well within a Mott shell with unity filling. The displayed error bars show the $1 \sigma$ statistical uncertainty, given by the Clopper-Pearson confidence limits. The data was fitted by a flat-top model function (see Appendix) and yields a fullwidth at half-maximum $\sigma_{a}=330(20) \mathrm{nm}$, an edge sharpness of $\sigma_{s}=50(10) \mathrm{nm}$, and a peak fidelity of $95(2) \%$. The offset was fixed at the $6(2) \%$ probability of thermally activated holes as deduced from the neighbouring and next neighbouring sites (blue shaded regions in $\mathbf{a}, \mathbf{b}$ and blue points in $\mathbf{c}$ ).

probability $p_{0}(\delta x)$ of finding an empty site as a function of the pointing offset $\delta x$ between the addressing beam and the center of the lattice site (see Fig.3p). We also investigated the effect of the addressing on neighbouring atoms, which ideally should remain unaffected. For this purpose, we monitored the probability of finding a hole at the sites next to the addressed ones (dark blue regions in Fig. $3 \mathrm{a}$, b and points in Fig. 3p). In order to distinguish accidentally flipped neighbouring atoms from holes that originate from thermal excitations of the initial Mott insulator [28, we also monitored the probability of finding a hole at the second next neighbours (light blue regions and points in Fig. 3). As both yielded the same hole probability of $6(2) \%$, we attribute all holes to thermal excitations and conclude that the probability of address- ing a neighbouring atom is indiscernibly small. We fitted the hole probability $p_{0}(\delta x)$ of the addressed site with a flat-top model function (see Appendix), keeping the offset fixed at the thermal contribution of $6 \%$. From the fit, we derived a spin-flip fidelity of $95(2) \%$, a full-width at half-maximum of $\sigma_{a}=330(10) \mathrm{nm}$ and an edge sharpness of $\sigma_{s}=50(10) \mathrm{nm}$ (Fig. 33 ). These values correspond to $60 \%$ and $10 \%$ of the addressing beam diameter, demonstrating that our method reaches sub-diffraction-limited resolution, well below the lattice spacing.

The observed maximum spin-flip fidelity is currently limited by the population transfer efficiency of our microwave sweep. The edge sharpness $\sigma_{s}$ originates from the beam pointing error of $\lesssim 0.1 a_{\text {lat }}$ and from variations in the magnetic bias field. The latter causes frequency fluctuations of $\sim 5 \mathrm{kHz}$, which translate into an effective pointing error of $0.05 a_{\text {lat }}$ at the maximum slope of the addressing beam profile. The resolution $\sigma_{a}$ could in principle be further reduced by a narrower microwave sweep, at the cost of a larger sensitivity to the magnetic field fluctuations. A larger addressing beam power would reduce this sensitivity, but we observed that this deformed the lattice potential due to the imperfect $\sigma^{-}$-polarization, allowing neighbouring atoms to tunnel to the addressed sites.

\section{Coherent tunneling dynamics}

The preparation of an arbitrary atom distribution opens up new possibilities for exploring coherent quantum dynamics at the single-atom level. As an example, we studied the tunnelling dynamics in a one-dimensional lattice (see Fig. 4) which allowed us to determine how much our addressing scheme affects the vibrational state of the atoms. We started by preparing a single line of up to 18 atoms along the $y$ direction before we lowered the lattice along the $x$ direction to $V_{x}=5.0(5) E_{r}$ within $200 \mu \mathrm{s}$. At the same time, the other lattices were lowered to $V_{y}=30 E_{r}$ and $V_{z}=23 E_{r}$, which reduced the external confinement along the $x$ direction, but still suppressed tunnelling in the $y$ and $z$ directions. After a varying hold time $t$, allowing the atoms to tunnel along $x$, the atomic distribution was frozen by a rapid $100 \mu \mathrm{s}$ ramp of all lattice axes to $70 E_{r}$. By averaging the resulting atomic distribution along the $y$ direction and repeating the experiment several times, we obtained the probability distribution of finding an atom at the different lattice sites (Fig. 4 bottom row).

This probability distribution samples the single-atom wave function after a coherent tunnelling evolution. We observed how the wave function expands in the lattice and how the interference of different paths leads to distinct maxima and minima in the distribution, leaving for example almost no atoms at the initial position after a single tunnelling time (Fig.4 4 ). This behaviour differs 

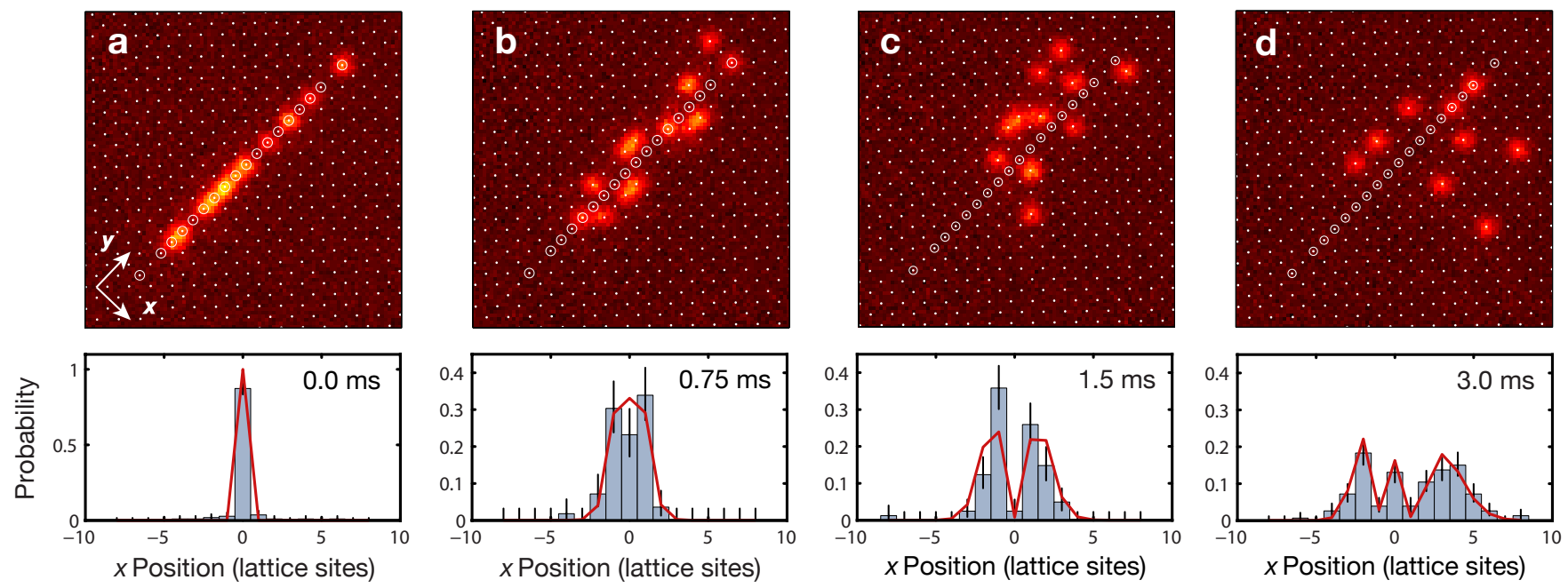

FIG. 4: Ground state tunnelling dynamics. a, Atoms were prepared in a single line along the $y$ direction before the lattice along the $x$ axis was lowered, allowing the atoms to tunnel in this direction (b-d). The top row shows snapshots of the atomic distribution after different hold times. White circles indicate the lattice sites at which the atoms were prepared (not all sites initially contained an atom). The bottom row shows the respective position distribution obtained from an average over $10-20$ of such pictures, the error bars give the $1 \sigma$ statistical uncertainty. A single fit to all distributions recorded at different hold times (red curve) yields a tunnelling coupling of $J^{(0)} / \hbar=940(20) \mathrm{Hz}$, a trap frequency of $\omega_{\text {trap }} /(2 \pi)=103(4) \mathrm{Hz}$ and a trap offset of $x_{\mathrm{offs}}=-6.3(6) a_{\text {lat }}$.

markedly from the evolution in free space, where a Gaussian wave packet disperses without changing its shape, always preserving a maximum probability in the center. For longer hold times, an asymmetry in the spatial distribution becomes apparent (Fig.44 $\mathrm{d}$ ), which originates from an offset between the bottom of the external harmonic confinement and the initial position of the atoms. We describe the observed tunnelling dynamics by a simple Hamiltonian including the tunnel coupling $J^{(0)}$ between two neighbouring sites and an external harmonic confinement, parameterized by the trap frequency $\omega_{\text {trap }}$, and the position offset $x_{\text {offs }}$ (see Appendix). A single fit to all probability distributions recorded at different hold times yields $J^{(0)} / \hbar=940(20) \mathrm{Hz}, \omega_{\text {trap }} /(2 \pi)=103(4) \mathrm{Hz}$ and $x_{\text {offs }}=-6.3(6) a_{\text {lat }}$. This is in agreement with the trap frequency $\omega_{\text {trap }} /(2 \pi)=107(2) \mathrm{Hz}$ obtained from an independent measurement via excitation of the dipole mode without the $x$ lattice, whose contribution to the external confinement is negligible compared to the other two axes. From $J^{(0)}$, we calculated a lattice depth of $V_{x}=4.6(1) E_{r}$, which agrees with an independent calibration via parametric heating. The expansion of the wave packet can also be understood by writing the initial localized wave function as a superposition of all Bloch waves of quasi-momentum $\hbar q$, with $-\pi / a_{\text {lat }} \leq q \leq$ $\pi / a_{\text {lat }}$. To each quasi-momentum $\hbar q$, one can assign a velocity $v_{q}=\frac{1}{\hbar} \frac{\partial E}{\partial q}$, determined by the dispersion relation $E(q)=-2 J^{(0)} \cos \left(q a_{\text {lat }}\right)$ of the lowest band. The edges of the wave packet propagate with the largest occurring velocity $v_{\max }=2 J^{(0)} a_{\text {lat }} / \hbar=1.88(4) a_{\text {lat }} / \mathrm{ms}$, in agreement with our data.

Our measurements constitute the first observation of the ground state tunnelling dynamics of massive particles on a lattice with single-site resolution. A similar continuous-time quantum walk has been demonstrated with photons in an array of evanescently coupled photonic waveguides [37. For massive particles, a discrete quantum walk of single atoms has been observed using a sequence of spin manipulations and spin-dependent transports in an optical lattice [38] and also with trapped ions [39. Without single-particle and single-site resolution, a continuous-time quantum walk in the ground state has been observed for ultracold fermionic atoms by measuring their ballistic expansion in a weak lattice [40].

In a second tunnelling experiment, we observed the faster dynamics of atoms in the first excited band (see Fig. 5p. For this, we deliberately excited the atoms by introducing a pointing offset $\delta x$ of the addressing beam, which caused a shift of the potential wells during the switch-on. We repeated the same tunnelling experiment as above with a hold time of $t=1 \mathrm{~ms}$ for different pointing offsets $\delta x$. For a small pointing offset $\left(\delta x=0.1 a_{\text {lat }}\right.$ in Fig.5p) we observed a narrow distribution, compared to a much broader one for a large offset $\left(\delta x=-0.4 a_{\text {lat }}\right.$ in Fig. 5 a). We attribute this to different fractions $f$ of atoms in the first band which is characterized by the higher tunnelling rate $J^{(1)}$. We fitted the distribution of Fig. 5 a to a two-band model (see Appendix) and found $J^{(1)} / \hbar=6.22(6) \mathrm{kHz}$. This is in excellent agreement with the expected value of $J^{(1)} / \hbar=6.14(6) \mathrm{kHz}$ from a band 

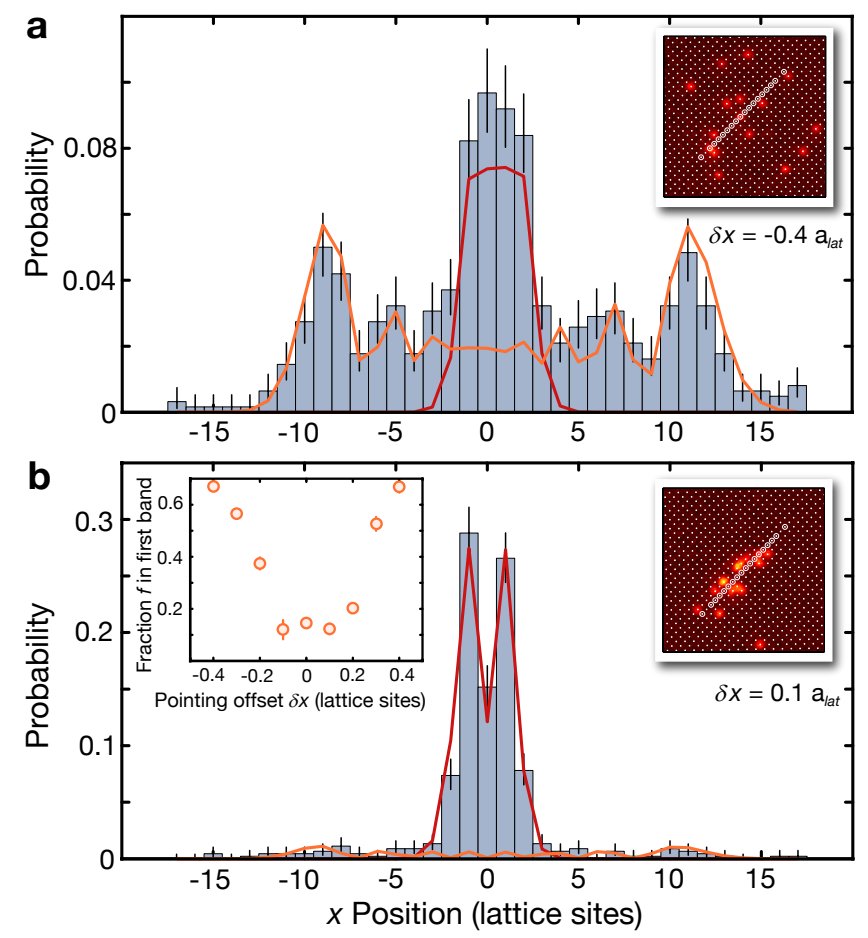

FIG. 5: Tunnelling dynamics of the first excited band. Some atoms were excited to the first band by a pointing offset $\delta x$ of the addressing beam. $\mathbf{a}$ and $\mathbf{b}$ show the atomic position distribution after $1 \mathrm{~ms}$ tunnelling time for $\delta x=-0.4 a_{\text {lat }}$ and $\delta x=0.1 a_{\text {lat }}$, respectively. We fitted the data with a model that includes atoms in the zeroth band (red line) and a fraction $f$ in the first band (orange line) (see Appendix). The right insets of $\mathbf{a}, \mathbf{b}$ show corresponding original images. The left inset of $\mathbf{b}$ shows $f$ versus $\delta x$ with a broad minimum of $f=13(2) \%$, indicating that most of the atoms are left in the ground state.

structure calculation in which we used $J^{(0)}$ as an input parameter to calculate the lattice depth. Our measurement of the fraction of excited atoms $f$ as a function of the pointing offset $\delta x$ (inset in Fig. 5 b) shows that the atoms are strongly heated for large pointing offsets. By contrast, only a small fraction of the atoms is excited to the first band for small pointing offsets $|\delta x| \leq 0.1 a_{\text {lat }}$, yielding a ground state population of $1-f=87(2) \%$.

\section{Discussion}

In summary, we have demonstrated full twodimensional single-site and single-atom spin control in an optical lattice with sub-diffraction-limited spatial resolution. Starting from a Mott insulator with unity filling, we achieved a spin-flip fidelity of 95(2)\% with negligible influence on the neighbouring lattice sites. Our scheme leaves most of the atoms in the vibrational ground state. The control of single spins in a strongly correlated many- body system on a lattice opens many new possibilities for studying quantum dynamics and quantum phases. Our technique will allow us to create out-of-equilibrium states or local perturbations in order to observe the ensuing dynamics of the many-body system, such as spin-charge separation [5, 6] or spin impurity dynamics beyond Luttinger liquid theory [4. Our studies of the tunnelling dynamics at the single-atom level can be extended to correlated particle tunnelling [41 43, also in higher dimensions, or to observe transport across local impurities 44 . or potential barriers. The tunneling can also be used to circumvent the pairwise losses during the imaging [27, 28] by letting the atoms of a one-dimensional system spread along the perpendicular direction in order to obtain a sufficiently low density. Further prospects are the implementation of novel cooling schemes relying on the local removal of regions with high entropy [8, 45]. The singlespin control in our large systems with several hundreds of atoms also opens new perspectives for scalable quantum computing. Combining single-qubit manipulation with local readout and a global entanglement operation in a spin-dependent lattice [46, 47] would be the basis of a one-way quantum computer 11, 12. For the circuit model of a quantum computer, two-qubit operations can be realised by Rydberg gates between selected atom pairs in the lattice [48, 49].

\section{Acknowledgements}

We thank Wolfgang Ketterle for stimulating discussions and valuable ideas. We acknowledge the help of Rosa Glöckner and Ralf Labouvie during the construction of the experiment. We acknowledge funding by MPG, DFG, Stiftung Rheinland-Pfalz für Innovation, Carl-Zeiss Stiftung, EU (NAMEQUAM, AQUTE, Marie Curie Fellowships to J.F.S. and M.C.), and JSPS (Postdoctoral Fellowship for Research Abroad to T.F.).

[1] G. Binnig and H. Rohrer, Rev. Mod. Phys. 59, 615 (1987).

[2] F. J. Giessibl, Rev. Mod. Phys. 75, 949 (2003).

[3] R. Blatt and D. Wineland, Nature 453, 1008 (2008).

[4] M. B. Zvonarev, V. V. Cheianov, and T. Giamarchi, Phys. Rev. Lett. 99, 240404 (2007).

[5] A. Recati, P. O. Fedichev, W. Zwerger, and P. Zoller, Phys. Rev. Lett. 90, 020401 (2003).

[6] A. Kleine, C. Kollath, I. P. McCulloch, T. Giamarchi, and U. Schollwöck, Phys. Rev. A 77, 013607 (2008).

[7] D. S. Weiss, J. Vala, A. V. Thapliyal, S. Myrgren, U. Vazirani, and K. B. Whaley, Phys. Rev. A 70, 040302(R) (2004).

[8] J.-S. Bernier, C. Kollath, A. Georges, L. De Leo, F. Gerbier, C. Salomon, and M. Köhl, Phys. Rev. A 79, 061601(R) (2009). 
[9] H. Weimer, M. Müller, I. Lesanovsky, P. Zoller, and H. P. Büchler, Nature Phys. 6, 382 (2010).

[10] M. A. Nielsen and I. L. Chuang, Quantum Computation and Quantum Information (Cambridge University Press, Cambridge, 2000).

[11] R. Raussendorf and H. J. Briegel, Phys. Rev. Lett. 86, 5188 (2001).

[12] H. J. Briegel, D. E. Browne, W. Dür, R. Raussendorf, and M. Van Den Nest, Nature Phys. 5, 19 (2009).

[13] R. Dumke, M. Volk, T. Müther, F. Buchkremer, G. Birkl, and W. Ertmer, Phys. Rev. Lett. 89, 097903 (2002).

[14] S. Bergamini, B. Darquié, M. Jones, L. Jacubowiez, A. Browaeys, and P. Grangier, J. Opt. Soc. Am. B 21, 1889 (2004).

[15] M. Saffman, Opt. Lett. 29, 1016 (2004).

[16] T. Calarco, U. Dorner, P. S. Julienne, C. J. Williams, and P. Zoller, Phys. Rev. A 70, 012306 (2004).

[17] C. Zhang, S. L. Rolston, and S. Das Sarma, Phys. Rev. A 74, 042316 (2006).

[18] J. Joo, Y. L. Lim, A. Beige, and P. L. Knight, Phys. Rev. A 74, 042344 (2006).

[19] J. Cho, Phys. Rev. Lett. 99, 020502 (2007).

[20] A. V. Gorshkov, L. Jiang, M. Greiner, P. Zoller, and M. D. Lukin, Phys. Rev. Lett. 100, 093005 (2008).

[21] N. Lundblad, J. M. Obrecht, I. B. Spielman, and J. V. Porto, Nature Phys. 5, 575 (2009).

[22] K. Shibata, S. Kato, A. Yamaguchi, S. Uetake, and Y. Takahashi, Appl. Phys. B 97, 753 (2009).

[23] R. Scheunemann, F. S. Cataliotti, T. W. Hänsch, and M. Weitz, Phys. Rev. A 62, 051801(R) (2000).

[24] D. Schrader, I. Dotsenko, M. Khudaverdyan, Y. Miroshnychenko, A. Rauschenbeutel, and D. Meschede, Phys. Rev. Lett. 93, 150501 (2004).

[25] M. Karski, L. Förster, J.-M. Choi, A. Steffen, N. Belmechri, W. Alt, D. Meschede, and A. Widera, New J. Phys. 12, 065027 (2010).

[26] P. Würtz, T. Langen, T. Gericke, A. Koglbauer, and H. Ott, Phys. Rev. Lett. 103, 080404 (2009).

[27] W. S. Bakr, A. Peng, M. E. Tai, R. Ma, J. Simon, J. I. Gillen, S. Fölling, L. Pollet, and M. Greiner, Science 329, 547 (2010).

[28] J. F. Sherson, C. Weitenberg, M. Endres, M. Cheneau, I. Bloch, and S. Kuhr, Nature 467, 68 (2010).

[29] N. Schlosser, G. Reymond, I. Protsenko, and P. Grangier, Nature 411, 1024 (2001).

[30] S. Kuhr, W. Alt, D. Schrader, M. Müller, V. Gomer, and D. Meschede, Science 293, 278 (2001).

[31] T. Grünzweig, A. Hilliard, M. McGovern, and M. F. Andersen, Nature Phys. 6, 951 (2010).

[32] M. P. A. Fisher, P. B. Weichman, G. Grinstein, and D. S. Fisher, Phys. Rev. B 40, 546 (1989).

[33] D. Jaksch, C. Bruder, J. I. Cirac, C. Gardiner, and P. Zoller, Phys. Rev. Lett. 81, 3108 (1998).

[34] M. Greiner, O. Mandel, T. Esslinger, T. W. Hänsch, and I. Bloch, Nature 415, 39 (2002).

[35] S. Fölling, A. Widera, T. Müller, F. Gerbier, and I. Bloch, Phys. Rev. Lett. 97, 060403 (2006).

[36] G. K. Campbell, J. Mun, M. Boyd, P. Medley, A. E. Leanhardt, L. Marcassa, D. Pritchard, and W. Ketterle, Science 313, 649 (2006).

[37] H. B. Perets, Y. Lahini, F. Pozzi, M. Sorel, R. Morandotti, and Y. Silberberg, Phys. Rev. Lett. 100, 170506 (2008).

[38] M. Karski, L. Förster, J.-M. Choi, A. Steffen, W. Alt,
D. Meschede, and A. Widera, Science 325, 174 (2009).

[39] F. Zähringer, G. Kirchmair, R. Gerritsma, E. Solano, R. Blatt, and C. F. Roos, Phys. Rev. Lett. 104, 100503 (2010).

[40] U. Schneider, L. Hackermüller, J. P. Ronzheimer, S. Will, S. Braun, T. Best, I. Bloch, E. Demler, S. Mandt, D. Rasch, et al., arXiv:1005.3545v1 (2010).

[41] K. Winkler, G. Thalhammer, F. Lang, R. Grimm, J. Hecker Denschlag, A. J. Daley, A. Kantian, H. P. Büchler, and P. Zoller, Nature 441, 853 (2006).

[42] S. Fölling, S. Trotzky, P. Cheinet, M. Feld, R. Saers, A. Widera, T. Müller, and I. Bloch, Nature 448, 1029 (2007).

[43] A. Peruzzo, M. Lobino, J. C. F. Matthews, N. Matsuda, A. Politi, K. Poulios, X.-Q. Zhou, Y. Lahini, N. Ismail, K. Wörhoff, et al., Science 329, 1500 (2010).

[44] A. Micheli, A. J. Daley, D. Jaksch, and P. Zoller, Phys. Rev. Lett. 93, 140408 (2004).

[45] J. F. Sherson and K. Mølmer, arXiv:1012.1457v1 (2010).

[46] D. Jaksch, H.-J. Briegel, J. I. Cirac, C. W. Gardiner, and P. Zoller, Phys. Rev. Lett. 82, 1975 (1999).

[47] O. Mandel, M. Greiner, A. Widera, T. Rom, T. Hänsch, and I. Bloch, Nature 425, 937 (2003).

[48] T. Wilk, A. Gaëtan, C. Evellin, J. Wolters, Y. Miroshnychenko, P. Grangier, and A. Browaeys, Phys. Rev. Lett. 104, 010502 (2010).

[49] L. Isenhower, E. Urban, X. L. Zhang, A. T. Gill, T. Henage, T. A. Johnson, T. G. Walker, and M. Saffman, Phys. Rev. Lett. 104, 010503 (2010).

[50] M. Garwood and L. DelaBarre, J. Magn. Reson. 153, 155 (2001).

\section{Appendix}

\section{Calibration of the addressing beam position}

To move the addressing laser beam in the object plane, we changed the angle of the beam entering from the reverse direction into the microscope objective using a twoaxis piezo mirror. The device has an angular resolution of $5 \mu \mathrm{rad}$, corresponding to a theoretical resolution in the object plane of $0.02 a_{\text {lat }} \simeq 10 \mathrm{~nm}$. In order to position the addressing laser beam onto the atoms with high precision, we measured calibration functions that translate the two control voltages of the piezo mirror into image coordinates. This calibration was performed by replacing the far detuned addressing laser beam by a near resonant molasses beam that follows the identical beam path. Using in addition the $x$ and $y$ molasses beams, we took a fluorescence image of a large thermal atom cloud in the vertical lattice alone and observed a strongly enhanced signal at the position of the focused beam. We determined the position of this fluorescence maximum with an uncertainty of 0.2 pixels in our images, corresponding to $0.05 a_{\text {lat }}=25 \mathrm{~nm}$ in the object plane. The long term drifts of the addressing beam position are on the order of $0.1 a_{\text {lat }}$ per hour, which we took into account by regular recalibration of the beam position. 


\section{Lattice phase feedback}

In order to compensate slow phase drifts of the optical lattice, we applied a feedback on the position of the addressing beam. We determined the two lattice phases along $x$ and $y$ after each realisation of the experiment by fitting the position of isolated atoms. Averaging over the positions of typically $1-5$ isolated atoms per image allowed us to determine the lattice phase to better than $0.05 a_{\text {lat }}$. For the determination of the phase, we used the lattice constant and the lattice angles determined from a fluorescence image with many isolated atoms 28. Since our phase drifts were slower than $0.04 a_{\text {lat }}$ between two successive realisations of the experiment ( $25 \mathrm{~s}$ cycle time), we used the lattice phase from the last image to correct the addressing beam position. This feedback was done by adding appropriate offsets to the piezo control voltages.

\section{Microwave sweeps}

Our microwave sweeps are HS1-pulses [50] with timedependent Rabi frequency $\Omega(t)$ and detuning $\delta(t)$ given by

$$
\begin{aligned}
\Omega(t) & =\Omega_{0} \operatorname{sech}\left[\beta\left(\frac{2 t}{T_{p}}-1\right)\right] \\
\delta(t) & =\frac{\sigma_{\mathrm{MW}}}{2} \tanh \left[\beta\left(\frac{2 t}{T_{p}}-1\right)\right],
\end{aligned}
$$

where $\Omega_{0} /(2 \pi)=3 \mathrm{kHz}$ is the maximum Rabi-frequency, $\beta=5$ is a truncation factor, $T_{p}=20 \mathrm{~ms}$ is the pulse length, and $\sigma_{\mathrm{MW}} /(2 \pi)=60 \mathrm{kHz}$ is the sweep width. The detuning $\delta(t)$ is measured relative to the center of the sweep at $\omega_{\mathrm{MW}}=\omega_{0}-\Delta_{\mathrm{MW}}$ (see Fig.1 $\mathrm{p}$ ). Here, $\omega_{0}$ is the bare resonance between the two hyperfine states, including the shift of $-570 \mathrm{kHz}$ due to the magnetic bias field along the $z$ direction and $\Delta_{\mathrm{MW}} /(2 \pi)=-75 \mathrm{kHz}$ denotes the offset of the sweep center.

\section{Spin-flip fidelity}

In order to determine the spin-flip fidelity, we fitted the hole probability $p_{0}$ as a function of the pointing offset $\delta x$ (see Fig. 3b) to a flat-top model function given by

$$
p_{0}(\delta x)=\frac{A}{2}\left[\operatorname{erf}\left(\frac{\delta x+\sigma_{a} / 2}{\sigma_{s}}\right)+\operatorname{erf}\left(-\frac{\delta x-\sigma_{a} / 2}{\sigma_{s}}\right)\right]+B .
$$

Here, $\operatorname{erf}(x)=2 / \sqrt{\pi} \int_{0}^{x} e^{-\tau^{2}} d \tau$ is the error function, $\sigma_{a}$ denotes the full-width at half-maximum of the flattop profile and $\sigma_{s}$ the edge sharpness. We chose this model function since our HS1-pulses (see above) produce a flat-top population transfer profile, the edges of which are dominated by randomly fluctuating quantities (beam pointing and magnetic fields) following Gaussian statistics. The addressing fidelity is defined as $F=A /(1-B)$ taking into account that the maximum hole probability $p_{0}^{\max }=A+B$ also includes holes from thermal defects.
These yield a hole with probability $B$ at unsuccessfully addressed sites which occur with probability $1-F$, such that $p_{0}^{\max }=F+(1-F) B$.

\section{Single-particle tunneling dynamics}

We describe the coherent tunneling dynamics on $k=$ $2 n+1$ lattice sites by the Hamiltonian

$$
\begin{aligned}
\hat{H}^{(0)}= & -J^{(0)} \sum_{i=-n}^{n}\left(\hat{a}_{i}^{+} \hat{a}_{i+1}+\hat{a}_{i}^{+} \hat{a}_{i-1}\right) \\
& +V_{\text {ext }} \sum_{i=-n}^{n}\left(i-x_{\text {offs }}\right)^{2} \hat{a}_{i}^{+} \hat{a}_{i},
\end{aligned}
$$

where $J^{(0)}$ is the tunnel coupling in the lowest band, $\hat{a}_{i}^{+}\left(\hat{a}_{i}\right)$ is the creation (annihilation) operator for a particle at site $i$. The strength of the external harmonic potential with trapping frequency $\omega_{\text {trap }}$ is given by $V_{\text {ext }}=\frac{1}{2} m \omega_{\text {trap }}^{2} a_{\text {lat }}^{2}$, and $x_{\text {offs }}$ describes a position offset with respect to the bottom of the harmonic potential. The single particle wave function and its coherent time evolution are given by

$$
\begin{aligned}
\Psi^{(0)}(t) & =\sum_{i=-n}^{n} c_{i}^{(0)}(t) \hat{a}_{i}^{+}|\tilde{0}\rangle \\
& =\exp \left(-i \hat{H}^{(0)} t / \hbar\right) \Psi^{(0)}(0),
\end{aligned}
$$

with the initial condition $\Psi^{(0)}(0)=\hat{a}_{0}^{+}|\tilde{0}\rangle$ and the vacuum state $|\tilde{0}\rangle$. The resulting probability of finding the particle at lattice site $i$ after time $t$ is $P_{i}^{(0)}(t)=\left|c_{i}^{(0)}(t)\right|^{2}$. For analyzing the data of Fig.4, we calculated the time evolution for $k=17$ lattice sites.

\section{Tunneling in the first excited band}

When including tunnelling in the first band, we assume an incoherent sum $P^{\text {tot }}(t)$ of the distributions $P^{(0)}(t)$ of the zeroth and $P^{(1)}(t)$ of the first band as

$$
P^{\text {tot }}(t)=(1-f) P^{(0)}(t)+f P^{(1)}(t) .
$$

The Hamiltonian $\hat{H}^{(1)}$ in the first band and the coherent dynamics are identical to the ones of the zeroth band (Eqs. 3 and 4), except for a different tunnel coupling $J^{(1)}$. When fitting this model to our data, we kept $\omega_{\text {trap }}, x_{\text {offs }}$ and $J^{(0)}$ fixed at the values obtained from the data displayed in Fig.4. We extracted $J^{(1)}$ from the data of Fig. $5 \mathrm{a}$ and used this value to fit the results for other pointing offsets. For the data in Fig. 5, the parameters of our microwave sweep were such that also neighbouring atoms were addressed. We took this into account by summing over two distinct probability distributions with a second starting position in the direction of the pointing offset. 